\title{
Factors That Influence Cascade-Induced Defect Growth in Pure Metals and Model Alloys
}

\author{
Toshimasa Yoshiie \\ Research Reactor Institute, Kyoto University, Kumatori-cho, Osaka-fu 590-0494, Japan
}

For the development of materials in a fusion reactor environment without a fusion reactor, it is important to understand the generation and accumulation of point defects. This paper discusses five important factors in metals that influence the initial stage of defect clustering under cascade damage conditions. The effect of the PKA energy spectrum on damage evolution was explained from the viewpoint of subcascade formation. Two origins of damage rate dependence of defect cluster formation, direct mutual annihilation of point defects and annihilation of point defects at cascade-induced defect clusters, were mentioned. As the effect of motion of interstitial clusters, an example was given to illustrate the strong correlation between the mobility of interstitial clusters and void growth. Thermal activation processes depend on irradiation temperature. Varying irradiation temperature experiment was evaluated as a technique for investigating the point defect processes during irradiation. Migration of alloying elements during irradiation was reviewed from the standpoint of the interaction of alloying elements with point defects.

(Received October 25, 2004; Accepted February 17, 2005)

Keywords: point defect clusters, neutron irradiation, void, cascade damage

\section{Introduction}

Materials irradiation experiments in fusion reactors are the best way to develop fusion reactor materials. In the absence of fusion reactors, materials irradiation tests with $14 \mathrm{MeV}$ neutrons are only possible using accelerators i.e., D-T fusion neutron sources. Irradiation data are scarce, however, especially at high doses, owing to the beam power of accelerators. Thus, irradiation experiments with fission neutrons, ions and electrons are used to study the defect structure evolution. For this, one needs to translate the data to the fusion neutron environment, which requires an understanding of factors that influence generation and accumulation of point defects. These factors include the primary knock-on atom (PKA) energy spectrum, damage rate, irradiation dose, irradiation temperatures, materials parameters such as crystal structures and mobility of defects, preexisting defects, and migration of alloying elements. Many studies have been conducted to clarify these factors. However, most of these were based on the so-called "conventional mean field approach", ${ }^{1)}$ and leaving out of the discussion on important features of cascade damage. The present paper focuses on the effects of five factors that influence defect clustering at the initial stage of irradiation based on results of recent studies on cascade damage by neutron irradiation.

\section{PKA Energy Spectrum}

The generation of PKAs is the first step of irradiation damage by neutrons in metals. A PKA, having acquired a high recoil energy, imparts its energy to the matrix, and thus induces a large cascade. In the cascade, a large number of vacancies become surrounded by interstitials. Vacancies, interstitials and their clusters formed in the cascade escape through intra-cascade reactions and start migrating in the matrix as freely migrating defects. These point defects and clusters play an important role in the defect structure evolution. A comarison between materials irradiation effects due to different irradiation fields requires a measure of actual defect formation. Displacement per atom (dpa) has been commonly used as the standard for measuring irradiation exposure in materials. It is defined as the average number of atoms displaced as a result of irradiation damage. One important deficiency in dpa modeling is insufficient treatment of cascade damage, especially for phenomena characteristic of high energy recoils, such as direct defect cluster formation in a cascade.

Figure 1 compares PKA energy spectra of fission versus fusion neutrons in Fe calculated using the SPECTER code. ${ }^{2)}$ Three irradiation fields of fission neutrons, namely the Japan Materials Testing Reactor (JMTR) of Japan Atomic Energy Research Institute, ${ }^{3)}$ the JOYO Mark II of Japan Nuclear Cycle Development Institute ${ }^{4}$ and the Kyoto University Research Reactor (KUR) of the Research Reactor Institute of Kyoto University ${ }^{5)}$ do not differ much. The average damage energy is defined as

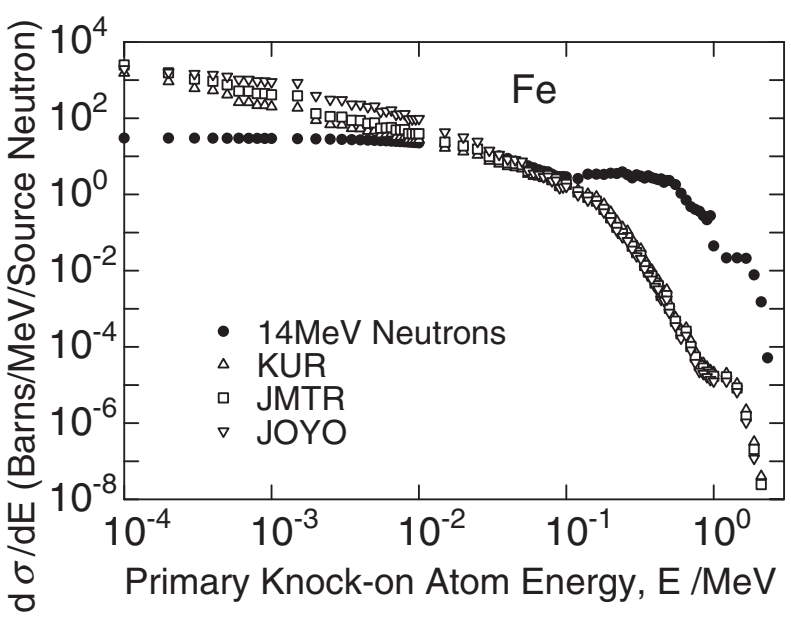

Fig. 1 Comparison of PKA energy spectra of Fe irradiated by fusion and fission neutrons in three irradiation fields, namely KUR, JMTR and JOYO. 
Table 1 The average damage energy in a fusion neutron irradiation field and three fission neutron irradiation fields.

\begin{tabular}{lcccc}
\hline \multirow{2}{*}{$\begin{array}{c}\text { Irradiation } \\
\text { Field }\end{array}$} & $\mathrm{Al}$ & $\mathrm{V}$ & $\mathrm{Fe}$ & $\mathrm{W}$ \\
\cline { 2 - 5 } & 111 & 144 & 151 & 71.1 \\
\hline $14 \mathrm{MeV}$ & 38.5 & 52.7 & 51.5 & 45.1 \\
JMTR & 43.2 & 57.5 & 56.8 & 45.5 \\
KUR & 33.1 & 49.0 & 48.2 & 45.2 \\
JOYO & & & & \\
\hline
\end{tabular}

$$
\int_{T_{\mathrm{TH}}}^{\infty} T(E) \frac{d \sigma(E)}{d E} d E / \int_{T_{\mathrm{TH}}}^{\infty} \frac{d \sigma(E)}{d E} d E,
$$

where $T_{\mathrm{TH}}$ is the displacement threshold energy, $T(E)$ is the damage energy and $\frac{d \sigma}{d E}$ is the differential cross-section of incident neutrons that collide to transfer a PKA energy $E$. We calculated the cross sections using the SPECTER code. ${ }^{2)}$ Table 1 compares average damage energy of $\mathrm{Al}, \mathrm{V}, \mathrm{Fe}$ and $\mathrm{W}$ in four irradiation fields. The variation in average damage energy between fission irradiation fields is about 1.3 fold. The damage energy of fusion neutrons, on the other hand, is several times higher than that of fission neutrons.

In order to model the effect of cascade damage, a PKA energy spectrum analysis has been proposed. ${ }^{6}$ ) This analysis is useful for comparing irradiation effects among different irradiation fields. The effect of PKA energy on defect cluster formation is determined by thin foil irradiation, where specimens are irradiated as electron microscopically observable thin foils. Surfaces are important sinks for freely migrating defects produced by irradiation, capturing most of the freely migrating defects near them. If stacking fault tetrahedra (SFTs) are formed directly in the cascades in fcc metals, and interstitials and their clusters escape to the surfaces in thin foil specimens, cascade structures are retained. At lower temperatures, the subcascade structure is observed. ${ }^{7}$ In the case of a compact cascade, such as those produced in $\mathrm{Au}$, with increasing irradiation temperature (above $473 \mathrm{~K}$ ) well-separated subcascades (SFTs) fuse into a large SFT. The SFT size increases with PKA energy. ${ }^{8)}$ Large SFTs are formed by cascade damage events, not annihilation of small SFTs. Results show that annealing of subcascade structures formed at $300 \mathrm{~K}$ irradiation does not lead to formation of large SFTs. ${ }^{9)}$ Figure 2 compares defect structures of fission (JMTR, $573 \mathrm{~K}, 0.044 \mathrm{dpa}$ ) versus fusion neutron irradiation (RTNS-II, $563 \mathrm{~K}, 0.017 \mathrm{dpa}$ ) as revealed by transmission electron microscopy (TEM). Though total displacement damage is higher for fission neutron irradiation, the remaining number of SFTs is higher in fusion neutron irradiation. The average size of SFTs is also larger in the case of fusion neutron irradiation. If there exists a threshold energy for SFT formation $\left(E_{\mathrm{TH}}\right)$, it can be evaluated using the following equation:

$$
N_{\mathrm{SFT}}=\alpha \varphi \int_{E_{\mathrm{TH}}}^{\infty} \frac{d \sigma(E)}{d E} d E,
$$

where $N_{\mathrm{SFT}}$ is the concentration of SFTs observed, $\alpha$ is the SFT formation efficiency and $\varphi$ is the total neutron fluence. PKA energy spectra $\frac{d \sigma}{d E}$ of Au calculated with the SPECTER code $^{2)}$ in two irradiation fields are shown in Fig. 3. $\alpha$ and $E_{\mathrm{TH}}$

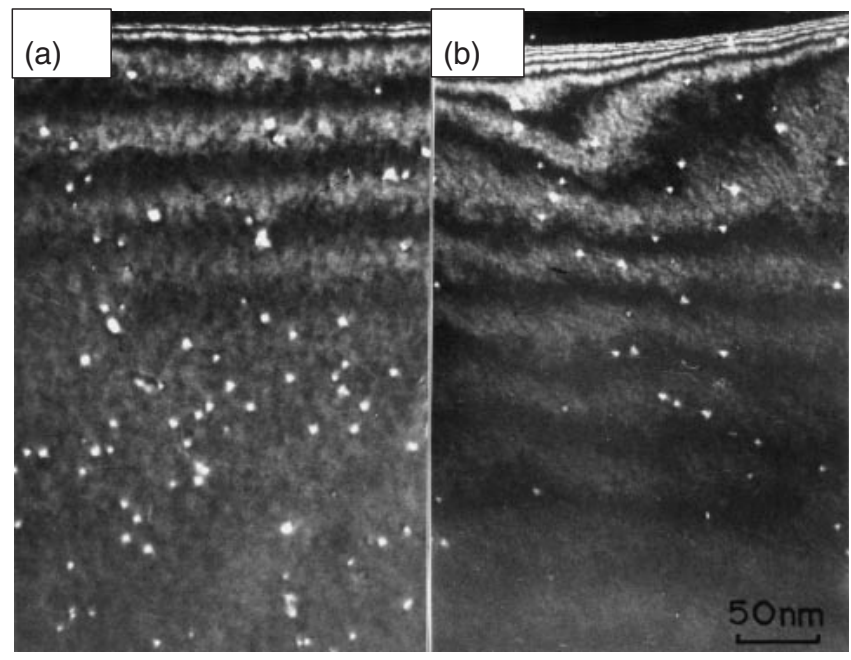

Fig. 2 Comparison of damage structures of (a) fusion neutrons (RTNS-II) $0.017 \mathrm{dpa}$ at $563 \mathrm{~K}$ and (b) fission neurons (JMTR) $0.044 \mathrm{dpa}$ at $573 \mathrm{~K}$ in thin foil irradiated $\mathrm{Au}$.

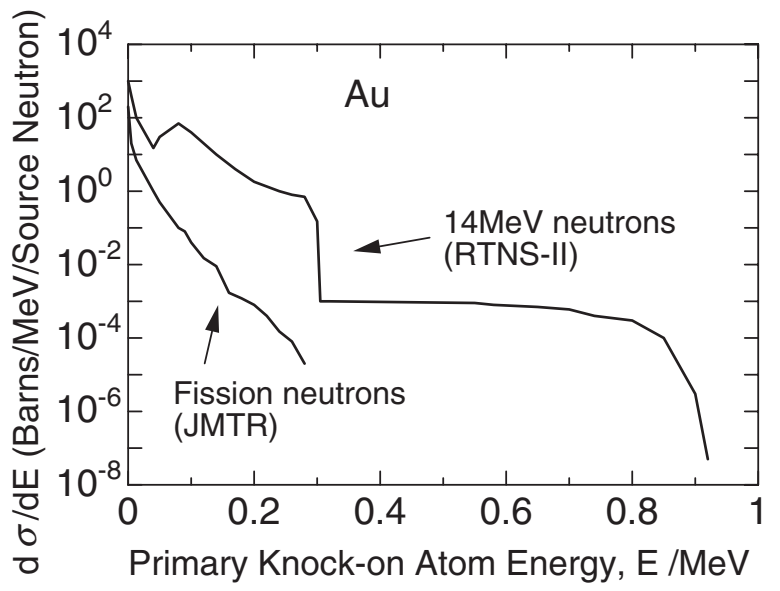

Fig. 3 Comparison of PKA energy spectra between fusion neutrons (RTNS-II) and fission neutrons (JMTR) in Au.

were estimated to be 0.05 and $80 \mathrm{keV}$, respectively, using the number density of SFTs formed by fission and fusion neutron irradiation,respectively. It is concluded that SFTs are formed for PKA energies larger than $80 \mathrm{keV}$ with a cascade efficiency of 0.05 at $563-573 \mathrm{~K}$.

For irradiation effects in metals with a medium atomic weight such as $\mathrm{Ni}, \mathrm{Cu}$ and $\mathrm{Fe}$, the unit dpa sometimes provides a good measure of defects formed by irradiation damage. This is because in such metals, medium cascades that are neither compact nor dilute are formed. When the damage energy is large, the cascade is divided into several subcascade areas. In compact cascades, the subcascades are very close together and they interact at higher temperatures, as in the example of Au mentioned above. In dilute cascades, the formation of subcascades is unclear. In medium cascades, the damaged area is divided into several well separated subcascades. Freely migrating defects and defect clusters are formed in each subcascade. Interactions between subcascades are not strong. The number of subcascades increases 


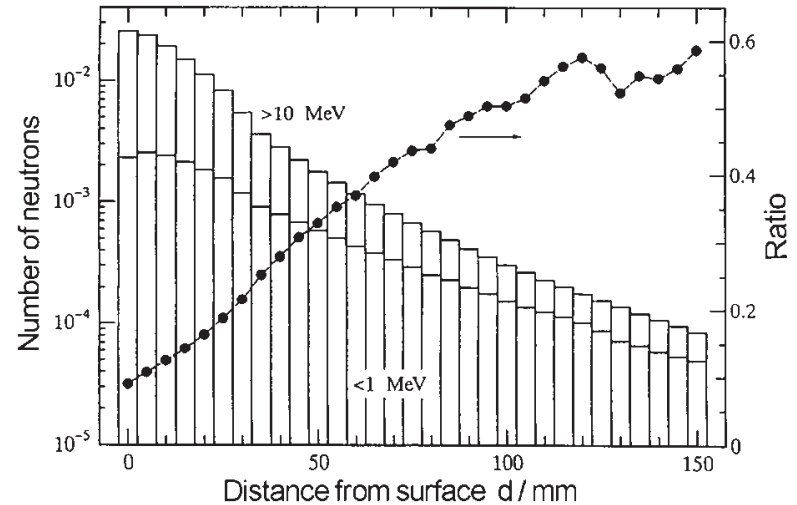

Fig. 4 Calculated change in the energy of $14 \mathrm{MeV}$ neutrons during transport in $\mathrm{Cu} .{ }^{10)}$ The stacked bar graph indicates the number of neutrons with energies lower than $1 \mathrm{MeV}$ (lower part) and higher than $10 \mathrm{MeV}$ (upper part). The line plot shows the ratio of the two groups. with the size of the original cascade. If the same number of freely migrating defects and nuclei of point defect clusters is generated from each subcascade in both fission and fusion neutron irradiated metals, the number of subcascades is important as a measure of damage structures. The number of subcascades is roughly obtained by dividing the damage energy by the subcascade formation threshold energy using the Kinchin-Pease model. Usually, the subcascade formation threshold energy of metals with a medium atomic weight is below $20 \mathrm{keV}$. In this case, the total displacement damage measured in dpa is proportional to the number of subcascades and the unit dpa is a good measure of the irradiation damage. ${ }^{8)}$

Ichihara et al. have measured and calculated the change of a $14 \mathrm{MeV}$ neutron spectrum during transport in metals. ${ }^{10,11)}$ Figure 4 shows the change of the neutron energy ratio above $10 \mathrm{MeV}$ and below $1 \mathrm{MeV}$ in $\mathrm{Cu}$. If $14 \mathrm{MeV}$ neutrons transport $10 \mathrm{~cm}$ of $\mathrm{Cu}$, the number of neutrons lower than $1 \mathrm{MeV}$ and higher than $10 \mathrm{MeV}$ is the same. For the precise analysis of defect structure evolution in metals irradiated by $14 \mathrm{MeV}$ neutrons, the variation in neutron spectrum should be considered.

\section{Migration of Defect Clusters}

Moving interstitial clusters were first observed in metals during electron irradiation in high voltage electron microscopy. ${ }^{12,13)}$ Movement of interstitial clusters was also often detected during post irradiation TEM observations of high energy particle irradiated metals. The formation of interstitial type dislocation loops near the dilatational side of edge dislocations ${ }^{14,15)}$ and alignment of these interstitial type dislocation loops along the $\langle 110\rangle$ directions ${ }^{16)}$ are the result of movement of interstitial clusters. Examples of these are shown in Figs. 5 and 6. The accumulation of loops near edge dislocations is only observed in a narrow temperature window. Below this window, many interstitial type dislocation loops are formed in the matrix, while no significant formation of loops is observed near edge dislocations. Above the window, the formation of interstitial type dislocation loops is difficult. By molecular dynamics simulation of

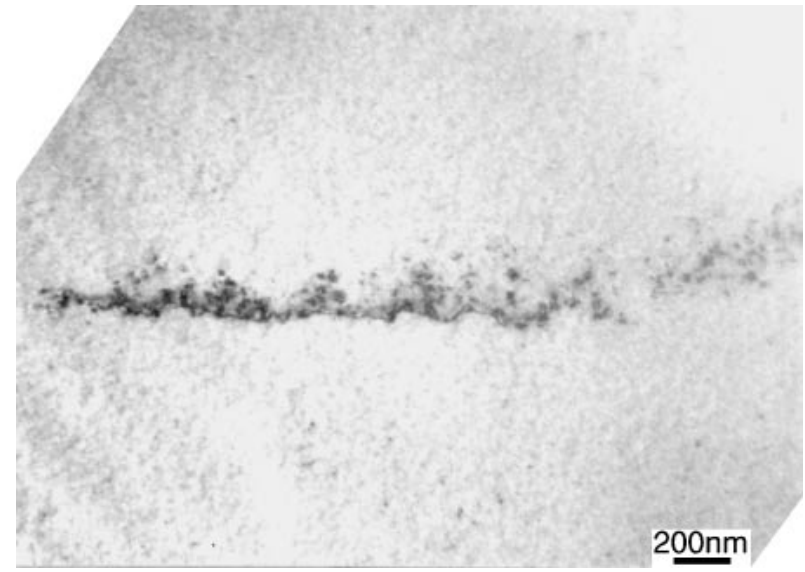

Fig. 5 Accumulation of interstitial type dislocation loops around an edge dislocation in fission neutron bulk irradiated Fe (JMTR) at $473 \mathrm{~K}$ to a dose of $0.062 \mathrm{dpa}$.

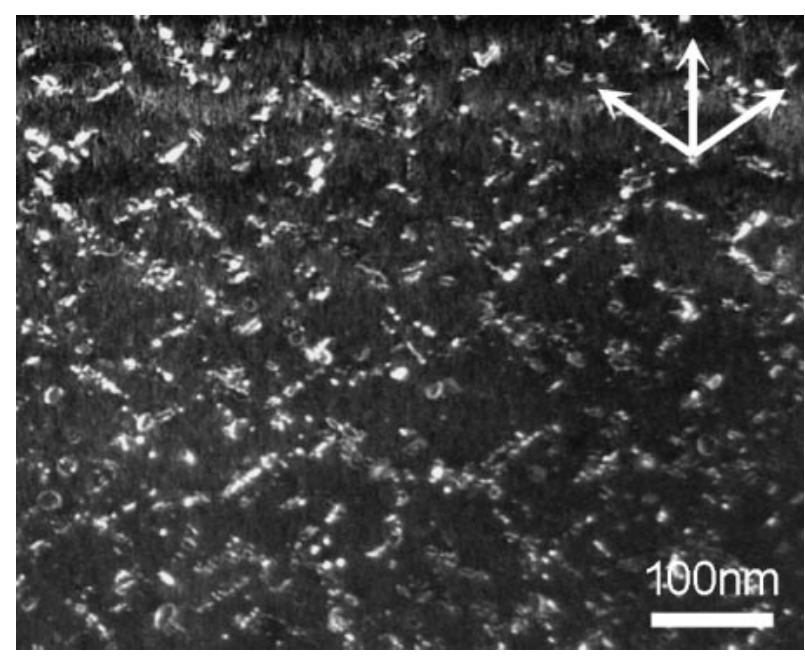

Fig. 6 Alignment of interstitial type dislocation loops in fission neutron thin foil irradiated $\mathrm{Ni}(\mathrm{JMTR})$ at $573 \mathrm{~K}$ to a dose of $0.13 \mathrm{dpa}$. Arrows indicate $\langle 110\rangle$ directions.

cascade damage, the one dimensional (1-D) motion of interstitial clusters was demonstrated. ${ }^{17)}$ Recently, direct insitu TEM of moving clusters in $\mathrm{Cu}$ during ion irradiation was performed. ${ }^{18)}$ The importance of this motion on defect structure evolution in metals has been pointed out by many researchers. ${ }^{13,19-21)}$ A recently proposed concept called "production bias", takes into account the formation and movement of interstitial clusters directly in cascades. ${ }^{1,20,21)}$ However, mobile clusters formed by the accumulation of single interstitials outside the cascade area also contribute to defect structure evolution since the reaction rate of interstitial clusters to other defects is lower than that of single interstitials.

Alloying elements are expected to affect the motion of interstitial clusters, and thus it is possible to investigate the role of the 1-D motion of interstitial clusters in microstructure evolution. Alloying elements in Ni change defect structures remarkably. ${ }^{14,22-24)}$ TEM revealed well developed dislocation networks and voids in neutron irradiated pure $\mathrm{Ni}$ 

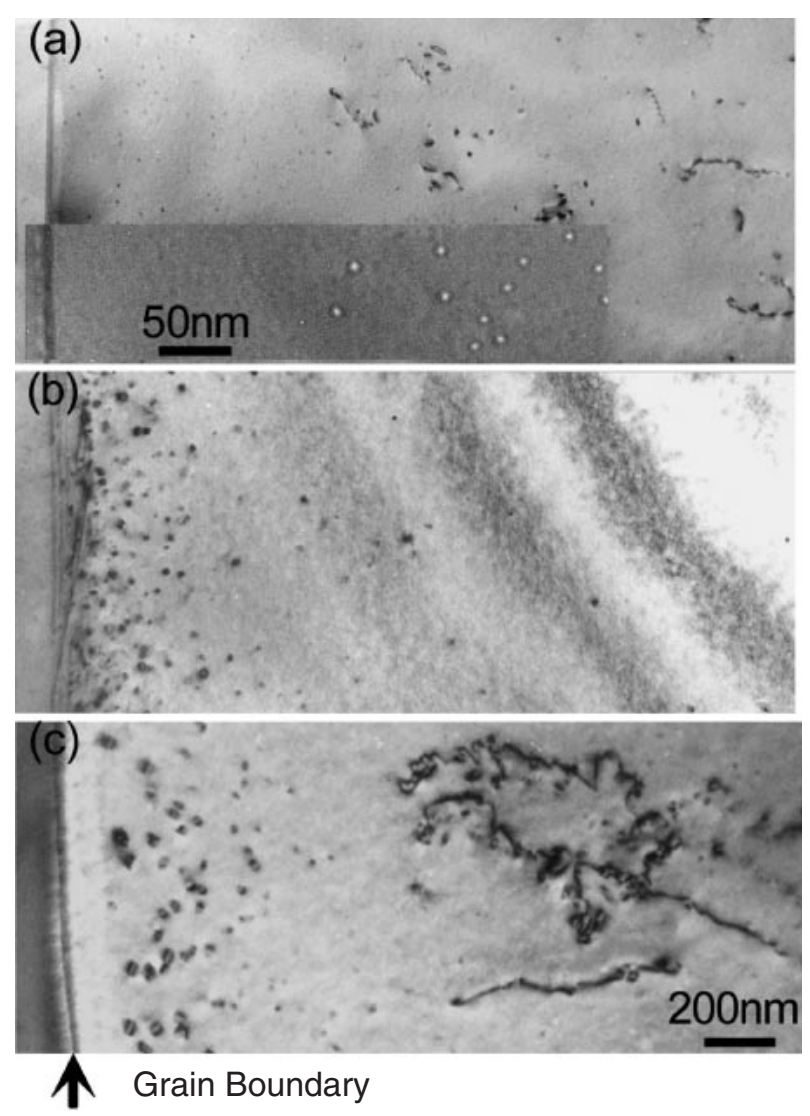

Fig. 7 Defect structures near grain boundaries of (a) $\mathrm{Ni}$ and (b) $\mathrm{Ni}-\mathrm{Sn}$ irradiated with improved temperature control to $0.13 \mathrm{dpa}$ and (c) $\mathrm{Ni}$ irradiated with conventional temperature control to $0.18 \mathrm{dpa}$ at the JMTR at $573 \mathrm{~K}$.

at $573 \mathrm{~K}$ above a dose of $0.026 \mathrm{dpa}$. The presence of microvoids was detected by positron lifetime measurement even at a low dose level of $0.001 \mathrm{dpa}^{24)}$ The addition of $2 \mathrm{at} \% \mathrm{Cu}$ (7.18\%: volume size factor to $\mathrm{Ni}^{25)}$ ) and $\mathrm{Ge}(14.76 \%)$ does not change the microstructure appreciably. On the other hand, after the addition of Si (-5.8\%) and Sn (74.08\%), no voids were detected by $\mathrm{TEM}^{23)}$ or positron lifetime measurement. ${ }^{24)}$ The defect structures in neutron irradiated $\mathrm{Ni}$ and Ni2 at $\%$ Sn to a dose of $0.13 \mathrm{dpa}$ at $573 \mathrm{~K}$ are shown in Fig. 7. Well developed dislocation structures and voids were observed in $\mathrm{Ni}$ as shown in Fig. 7(a). The micrograph insert in (a) was taken with the so-called void contrast condition. On the other hand, interstitial type dislocation loops were observed preferentially near grain boundaries in $\mathrm{Ni}-\mathrm{Sn}$ as shown in Fig. 7(b). The loops were found on each of the four $\{111\}$ planes with almost the same ratio.

These results were obtained by employing improved temperature control irradiation, where the specimen temperature was kept at the irradiation temperature before reactor startup to avoid a temperature history. ${ }^{26}$ ) In the case of conventional irradiation, where specimen temperature changes with reactor power, loops were observed near the grain boundary, in addition to the voids and dislocation structures in the matrix as shown in Fig. 7(c). These loops are thought to be formed by irradiation during the temperature transient of conventional irradiation.

A reaction kinetics analysis that included the point defect

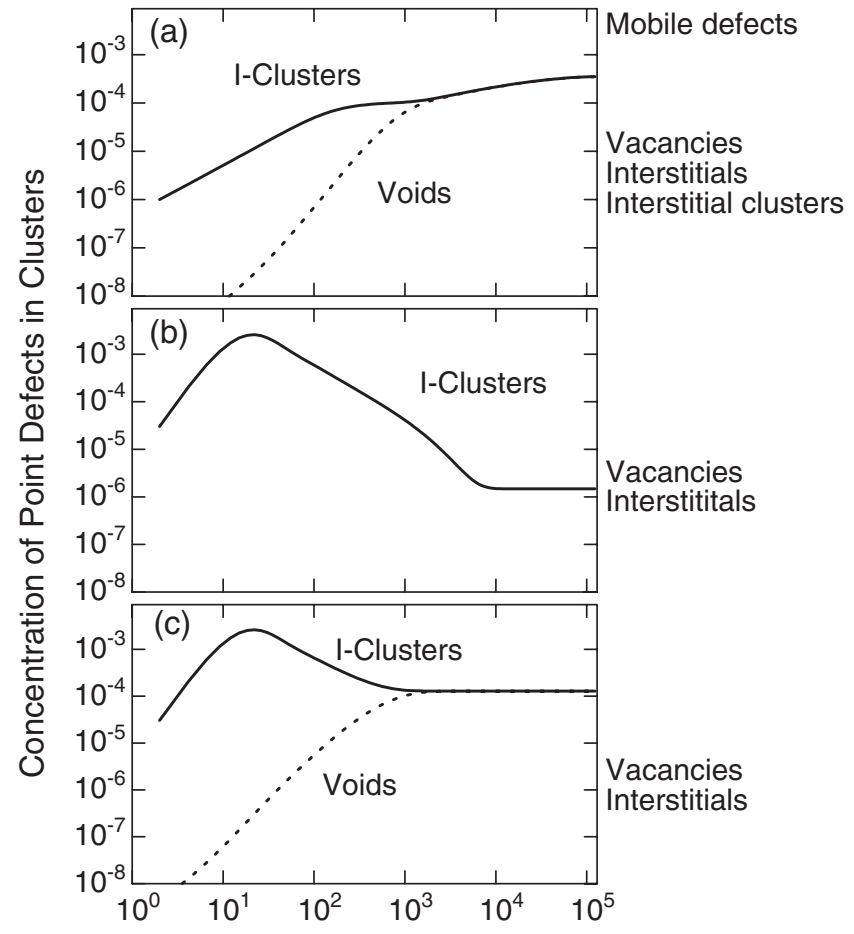

Distance from Planar Sink, d/ atomic distance

Fig. 8 Simulation of defect accumulation near grain boundaries to a dose of $0.2 \mathrm{dpa}$ in Ni at $573 \mathrm{~K}$. (a), (b) and (c) correspond to Figs. 7(a), (b) and (c), respectively.

flow into the planar sink (surface or grain boundary) was performed with and without 1-D motion of point defect clusters. ${ }^{8)}$ Figure 8 shows the results for three different systems. The concentration of point defects and their clusters was assumed to be 0 at the planar sink. In a system with interstitial clusters (dislocation loops) and voids, if interstitial clusters, like point defects, are mobile, voids and interstitial clusters grow in the matrix and the growth of interstitial clusters near the planar sink is suppressed, as shown in Fig. 8(a). In such a system, the growth of interstitial clusters leads to accumulation of excess vacancies in voids in the matrix.

In a system in which interstitial clusters are able to grow but are immobile, they grow only in a narrow volume near the planar sink as shown in Fig. 8(b). In such a system, vacancies accumulated in the matrix are spent to annihilate interstitial clusters and few interstitial clusters exist. In a system in which interstitial clusters and voids co-exist with no migration of interstitial clusters, interstitial clusters accumulate near the grain boundary, as shown in Fig. 8(c). As the defect structure in Fig. 7(a) was similar to that in Fig. 8(a), rather than to that in Fig. 8(c), it was concluded that in a system with both voids and interstitial clusters, interstitial clusters exhibit 1-D motion. The fact that the defect structure in Fig. 8(b) was identical to that in Fig. 7(b) indicates that interstitial clusters do not show 1-D motion in a system with no void growth.

The effect of alloying elements on void growth has been explained by the interaction with point defects. Oversized elements such as $\mathrm{Sn}$ in Ni will interact with vacancies and delay void growth. If alloying elements affect the 1-D motion 
of interstitial clusters, another possible explanation for the effect of alloying elements such as $\mathrm{Sn}$ in $\mathrm{Ni}$ is that they trap interstitial clusters and prevent their motion. Okazawa et al. detected interstitial clusters near the oversized element Hf in $\mathrm{Ni}$ using perturbed angular correlation measurements and positron annihilation lifetime measurements. ${ }^{27)}$

Another important result was the temperature dependence of 1-D motion. A good agreement was obtained between Figs. 7(c) and 8(c). The accumulation of interstitial clusters near grain boundaries evidenced the absence of 1-D motion, and consequently, of void growth. Voids, however, do grow under these irradiation conditions at $573 \mathrm{~K}$ in Ni. This is explained by the deficiency of conventional irradiation. Interstitial clusters near grain boundaries must form during irradiation at lower temperatures. This suggests to us that below $573 \mathrm{~K}$, say $473 \mathrm{~K}$ in Ni, the 1-D motion of interstitial clusters is insignificant. The migration energy of this motion is very low, lower than that of a single interstitial when the cluster contains less than 100 interstitials in Ni. ${ }^{19)}$ Thus, no temperature dependence is expected above room temperature. Moreover, if clusters move with the frequency of lattice vibration and the activation energy is lower than $0.1 \mathrm{eV}$, it is impossible to observe their movement using TEM. The movement has, however, been observed by many researchers. ${ }^{12,13,18)}$ The effect of $\mathrm{C}$ in $\mathrm{Cu}$ on the diffusion rate of point defect clusters was discussed by Abe et al. ${ }^{18)}$ The actual long range migration of interstitial clusters may be strongly affected by impurities and stress fields near defects.

For the movement of vacancy clusters, the migration of bubbles is well known. ${ }^{28)}$ Recently, the movement and splitting of voids during annealing were reported by Mukouda et al. ${ }^{29)}$ Figure 9 shows an example of void movement at $523 \mathrm{~K}$. The $\mathrm{Cu}$ specimen was irradiated at $573 \mathrm{~K}$ to $3.0 \times 10^{-4} \mathrm{dpa}$ at the KUR. The annealing temper- ature was relatively low. Void growth by coalescence of mobile voids during irradiation is probably one of the growth mechanisms far below the evaporation temperature.

\section{Damage Rate}

Structural materials of fission and fusion reactors are designed to last for several decades. However, irradiation tests can not continue this long, and thus, accelerated irradiation tests with high damage rates are performed. In order to ensure the long term safety of nuclear power plants, the effect of damage rate on defect structure evolution needs to be well understood. The overlap of component processes such as the lifetime of point defects and their clusters is a key issue in this regard. A basic analysis was performed by Kiritani. ${ }^{30)}$

Okita et al. estimated the effect of damage rate on neutroninduced microstructure evolution experimentally. ${ }^{31)}$ The net flux, the difference between vacancy and interstitial flux, was found to be proportional to $(\mathrm{dpa} / \mathrm{s})^{0.5}$ up to $28.8 \mathrm{dpa}$. They concluded that the mutual annihilation of point defects was the origin, even though point defect sinks such as cavities and dislocations were well developed.

Yanagita et al. studied the damage rate dependence of $\mathrm{Fe}-$ $\mathrm{Cu}$ alloys, taking into the effect of cascade damage in their analysis. Alloys were irradiated at $573 \mathrm{~K}$ under two different damage rates using the Hydraulic Conveyor (Hyd: $5.0 \times$ $\left.10^{-8} \mathrm{dpa} / \mathrm{s}\right)$ and the Slant Exposure Tube (Sl: $3.8 \times 10^{-10}$ $\mathrm{dpa} / \mathrm{s})$ in the KUR. ${ }^{32}$ The results of positron lifetime measurements and mechanical property tests clearly indicated a damage rate dependence. Microvoid formation and mechanical property change were larger in irradiation at lower damage rates. Figure 10 compares hardness change as a function of $\mathrm{Cu}$ concentration at high versus low damage
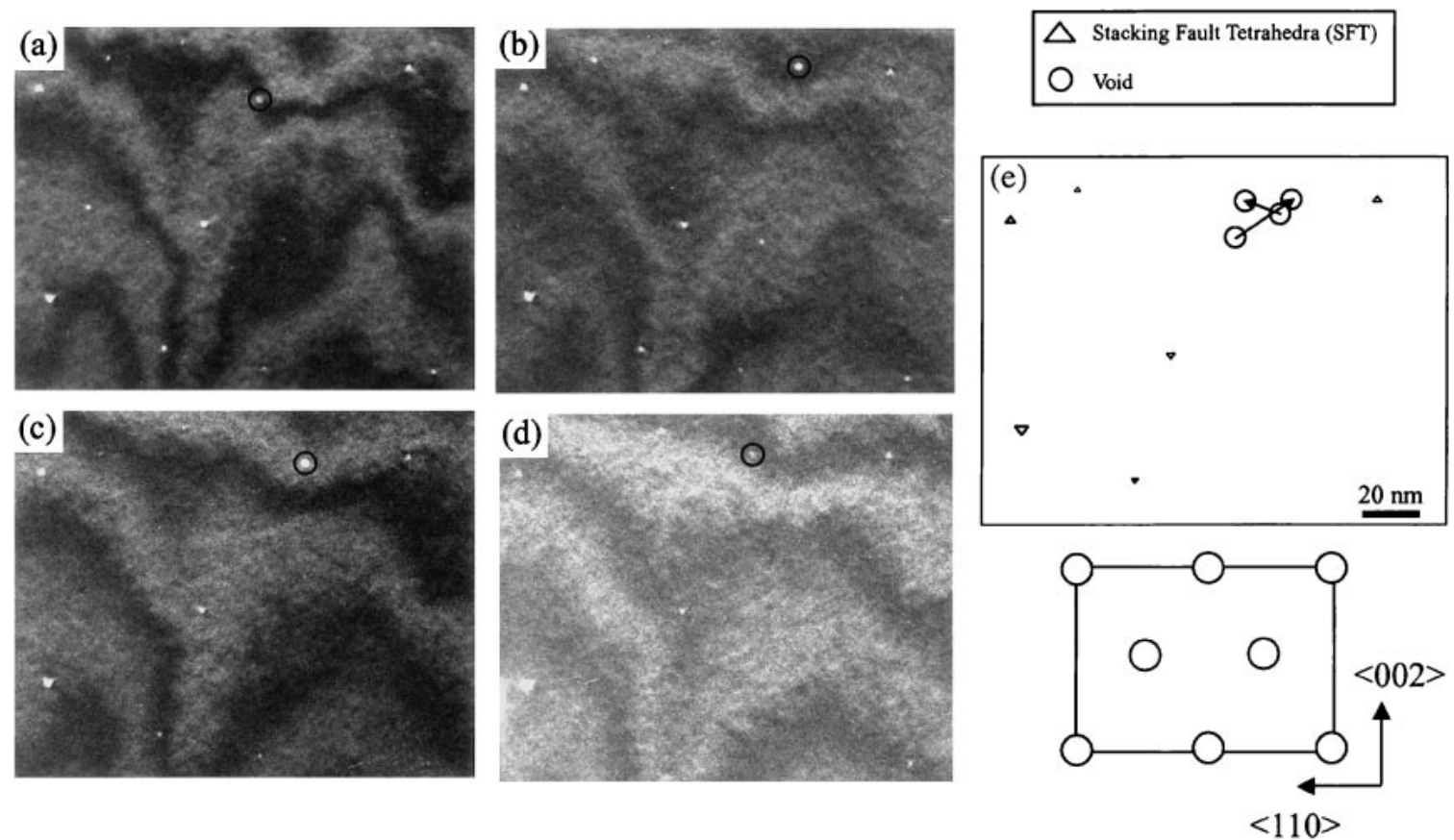

Fig. 9 Change of annealing step of $\mathrm{Cu}$, neutron irradiated at the KUR to $3.0 \times 10^{-4} \mathrm{dpa}$ at $573 \mathrm{~K}$. (a) $523 \mathrm{~K}, 0 \mathrm{~min}$, (b) $523 \mathrm{~K}, 10 \mathrm{~min}$, (c) $523 \mathrm{~K}, 30 \mathrm{~min}$, (d) $523 \mathrm{~K}, 60 \mathrm{~min}$ and (e) a schematic of this region. ${ }^{29}$ ) 


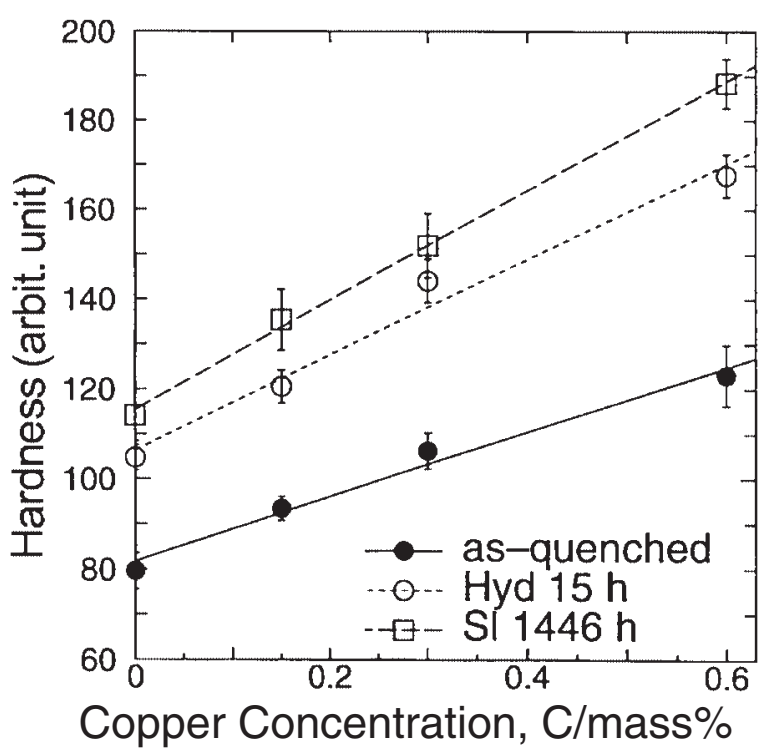

Fig. 10 Variation in micro Vickers hardness of neutron irradiated Fe0.6 mass $\% \mathrm{Cu}$ at $573 \mathrm{~K}$ with a high damage rate of $5.0 \times 10^{-8}$ to $2.9 \times$ $10^{-3} \mathrm{dpa}$ with Hyd and a low damage rate of $3.8 \times 10^{-10}$ to $2.2 \times 10^{-3}$ dpa with S1.

rate. The model by Yanagita et al. was formulated through rate equations. The rate of direct mutual annihilation between interstitials and vacancies was very low because of the low production rate of point defects in both irradiation fields. They believed that the point defect clusters that nucleated directly as a result of cascade damage were unstable at the irradiation temperature they used. Thus, they argued that the annihilation of point defects at the nuclei was the main mechanism for damage rate dependence. ${ }^{33,34)}$ At the lower damage rate, the nuclei evaporated before they could absorb point defects, and point defects promoted the growth of larger defect clusters. At the higher damage rate, point defects were absorbed by nuclei before they could evaporate; the nuclei acted as an effective sink for the point defects.

\section{Irradiation Temperature}

Irradiation temperature is one of most important factors controlling damage structure evolution; it greatly influences thermal activation processes. At low temperatures, nucleation of point defect clusters is easy. When the irradiation fluence is low, it is possible to observe the defect clusters formed directly in a cascade. ${ }^{7)}$ At high temperatures, point defect clusters evaporate and aggolomerate. Figures 11(a)(c) show the temperature dependence of defect structures in bulk irradiated $\mathrm{Ni}^{35)}$ At low temperatures, stacking fault tetrahedral and small interstitial type dislocation loops are observed. At high temperatures (above $573 \mathrm{~K}$ ), voids and well developed dislocation structures formed by the growth of loops are observed. Interstitial type dislocation loops near edge dislocations (see section 3) appear under irradiation at around $573 \mathrm{~K}$, as shown in Fig. 11(b).

The mixture of low and high temperature irradiation induces complex effects in defect structure evolution. Kiritani pointed out to the infeasibility of specimen temperature control in conventional irradiation by fission reactors. ${ }^{36)}$ In most cases in the past, specimens were heated using nuclear heating during irradiation. Thus, prior to reactor startup, the specimen was at the same temperatures as the coolant, and only at full power operation was the desired specimen temperature reached. Improved temperature control irradiation was first performed in the JMTR in $1988 .{ }^{26)}$ Specimens were designed to remain at the desired temperature using electric heaters, i.e., independently of reactor power. Ever since, improved temperature control has become a standard in fission neutron irradiation. ${ }^{5,37)}$

As the temperature during irradiation could be changed arbitrarily, temperature cycle irradiation runs were performed in the JMTR. ${ }^{38)}$ The original aim was to investigate the effect of temperature history on materials irradiation tests and determine the reliability of previous data that included the temperature history. Later the main aim became to predict the performance of fusion structural materials subject to temperature history during operation. ${ }^{39)}$ Irradiation experi-
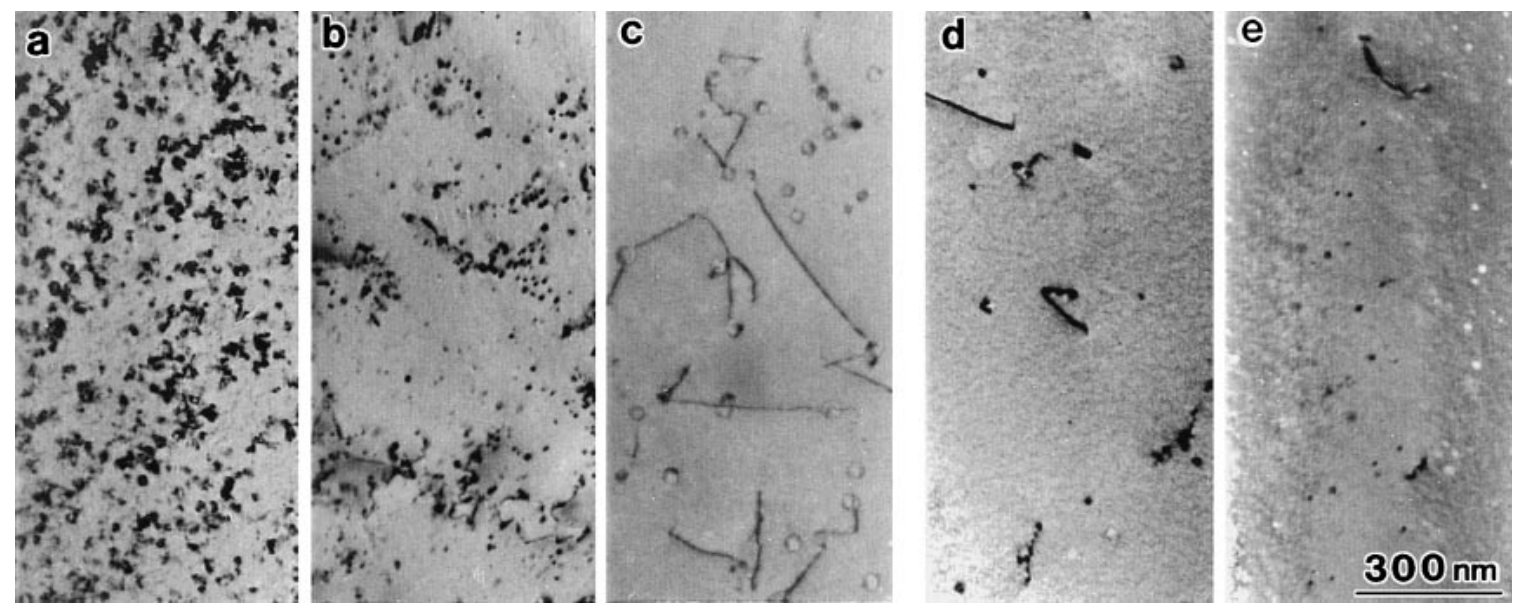

Fig. 11 Change of damage structures in fission neutron bulk irradiated $\mathrm{Ni}$ as a function of irradiation temperature. Constant temperature irradiation at (a) $473 \mathrm{~K}$ to $0.077 \mathrm{dpa}$, (b) $573 \mathrm{~K}$ to $0.11 \mathrm{dpa}$ and (c) $673 \mathrm{~K}$ to $0.3 \mathrm{dpa}$. Six temperature cycle irradiation at (d) $473 / 673 \mathrm{~K}$ to $0.26 \mathrm{dpa}$ and (e) $573 / 673 \mathrm{~K}$ to $0.43 \mathrm{dpa}$. 
ments at varing temperatures were performed in the HFIR in the framework of the Japan-USA Fusion Cooperation Program (JUPITER project) to understand the effect over a wide range from $0.1 \mathrm{dpa}$ (JMTR) to $5 \mathrm{dpa}$ (HFIR). ${ }^{40)}$

Now this irradiation technique is also recognized as an important method for verifying point defect processes during irradiation. As shown in Figs. 11(d) and (e), irradiation at $473 / 673 \mathrm{~K}$ yields a lower void density and irradiation at 573/ $673 \mathrm{~K}$ yields higher void density than that obtained by constant irradiation at $673 \mathrm{~K}$. Yoshida et al. studied the effect of temperature cycles in model alloys of austenitic stainless steels in the $473 / 673 \mathrm{~K}$ and $573 / 723 \mathrm{~K}$ range. In $\mathrm{Fe}-16 \mathrm{Cr}$ $17 \mathrm{Ni}-0.1 \mathrm{P}$, irradiation defects decreased, while in $\mathrm{Fe}-16 \mathrm{Cr}$ $17 \mathrm{Ni}-0.25 \mathrm{Ti}$, the defect structure evolution was accelerated. ${ }^{41)}$ In both cases, precise analysis revealed the stability of point defect clusters formed at lower temperatures. As the temperature transient can substantially alter damage structures, this method has the potential to reduce damage accumulation in reactors by only changing their thermal history during operation.

\section{Migration of Alloying Elements}

Solute atoms in alloys interact with point defects produced by irradiation damage, which leads to the segregation of alloying elements to sinks of point defects and the formation of precipitates in the matrix. The decrease of alloying element content in the matrix affects cascade events as well as migration of point defects. For example, alloying elements vary the cascade size by changing the length of the replacement collision sequence. ${ }^{42)}$

Recently, with the development of experimental techniques such as 3D atom prove and positron annihilation spectroscopy, precise atomistic level data has been obtained. Nagai et al. have shown the formation of voids and subsequent growth of $\mathrm{Cu}$ precipitates in voids in $\mathrm{Fe}$ 0.6 mass $\% \mathrm{Cu}^{43)} \mathrm{Xu}$ et al. detected an initial precipitation of $\mathrm{Cu}$ clusters at an early stage of irradiation prior to void formation. ${ }^{44)}$ The synergetic effect, the concurrent formation of vacancy clusters and precipitates, however, is not fully understood.

Most alloying elements move with the help of vacancies. Undersized elements are able to move with interstitials as mixed dumbbells. A recent study by Onitsuka et al. points to the $\mathrm{Cu}$ precipitation in a $\mathrm{Fe}-\mathrm{Cu}$ alloy by the contribution of interstitials below stage III. $^{45)}$ Since mixed dumbbells typically form with undersized elements, it has been believed that the formation of mixed dumbbells is difficult in the $\mathrm{Fe}-$ $\mathrm{Cu}$ system. For example, according to King, $\left.{ }^{25}\right) \mathrm{Cu}$ is an oversized element in Fe and the volume size factor is $17.53 \%$. The result of experimental measurements by Wriedt et al. was $11.6 \% .{ }^{46)}$ Ackland et al. calculated the factor using a set of many-body interatomic potentials and obtained $8.76 \% .{ }^{47}$ ) The atomic volume of $\mathrm{Cu}$ in bcc $\mathrm{Cu}$ precipitates in $\mathrm{Fe}$ formed by electron irradiation at $573 \mathrm{~K}$ was about $2 \%$ larger than that of $\mathrm{Fe}^{48)}$ The result of $a b$ initio calculations was 4.03 or $5.03 \%$ depending on the method used. ${ }^{49)}$ The volume size factor is an important parameter for alloy design. Urgent determination of such basic data is required.

\section{Closing Remarks}

Recent advances in our understanding of defect structural evolution in metals were presented. The studies cited in this paper used only pure metals and model alloys to simplify the analysis. There exists a wide gap between model alloys and practical alloys in terms of the generation and accumulation of point defects. For example, it is still unclear whether or not 1-D motion of interstitial clusters occurs in ferritic/martensitic steels. Minor elements in practical alloys will affect the mobility of migrating defects and the stability of defect clusters. The application of this knowledge to practical alloys is essential.

It is important to note that the role of the factors discussed in the present paper is not clear at high cluster densities. The development of fusion reactor materials requires an understanding of the physical features of cascade damage under high irradiation dose.

\section{Acknowledgements}

A part of this work was performed at the International Research Center for Nuclear Materials Science, Institute for Materials Research, Tohoku University.

\section{REFERENCES}

1) B. N. Singh, H. Trinkaus and C. H. Woo: J. Nucl. Mater. 212-215 (1994) 168.

2) L. R. Greenwood and R. K. Smither: ANL/FPP/TM-197, Argonne National Laboratory, 1985.

3) JAERI-DATA/Code 99-043, Japan Atomic Energy Research Institute.

4) JNC TN9450 2002-005, Japan Nuclear Cycle Development Institute.

5) T. Yoshiie, Y. Hayashi, S. Yanagita, Q. Xu, Y. Satoh, H. Tsujimoto, T. Kozuka, K. Kamae, K. Mishima, S. Shiroya, K. Kobayashi, M. Utsuro and Y. Fujita: Nucl. Instrum. Methods Phys. Res., Sect. A 498 (2003) 522-531.

6) M. Kiritani, T. Yoshiie, S. Kojima, Y. Satoh and K. Hamada: J. Nucl. Mater. 174 (1990) 327-351.

7) M. Kiritani, T. Yoshiie, S. Kojima and Y. Satoh: Radiation Effect. Defect. Solid. 113 (1990) 75-96.

8) T. Yoshiie, Y. Satoh and Q. Xu: J. Nucl. Mater. 329-333 (2004) 81-87.

9) Y. Shimomura, H. Fukushima, M. Kami, T. Yoshiie, H. Yoshida and M. Kiritani: J. Nucle. Mater. 141-143 (1986) 846.

10) C. Ichihara: Proc. Workshop on Development and Installation of an Irradiation Facility with Improved Control Capability and the Study of Materials Irradiation Effects 1994 (Research Reactor Institute, Kyoto University) p. 38-44.

11) C. Ichihara, I. Kimura, S. A. Hayashi, J. Yamamoto and K. Takahashi: J. Nucl. Sci. Technol. 40 (2003) 417-422.

12) M. Kiritani: Sixth Int. Conf. High. Voltage Electron Microscopy. Antwerp 1980, 196-203.

13) M. Kiritani: J. Nucl. Mater. 251 (1997) 237-251

14) S. Kojoma, T. Yoshiie and M. Kiritani: J. Nucl. Mater. 155-157 (1988) 1249-1253.

15) M. Kiritani, T. Yoshiie, S. Kojima and Y. Satoh: J. Nucl. Mater. 205 (1993) 460-466.

16) T. Yoshiie, S. Kojima and M. Kiritani: J. Nucl. Mater. 212-215 (1994) 186-191.

17) A. J. E. Foreman, C. A. English and W. J. Phythian: Philos. Mag. A 66 (1992) 655-669.

18) H. Abe, N. Sekimura and Y. Yang: J. Nucl. Mater. 323 (2003) 20-228.

19) E. Kuramoto: J. Nucl. Mater. 276 (2000) 143-153.

20) B. N. Singh, H. Trinkaus and C. H. Woo: J. Nucl. Mater. 212-215 (1994) 168-174.

21) S. I. Golubov, B. N. Singh and H. Trinkaus: J. Nucl. Mater. 276 (2000) 
$78-89$.

22) T. Yoshiie, S. Kojima, Y. Satoh, K. Hamada and M. Kiritani: J. Nucl. Mater. 191-194 (1992) 1160-1165.

23) K. Hamada, S. Kojima, Y. Ogasawara, T. Yoshiie and M. Kiritani: J. Nucl. Mater. 212-215 (1994) 270-274.

24) T. Yoshiie, Q. Xu, Y. Satoh, H. Ohkubo and M. Kiritani: J. Nucl. Mater. 283-287 (2000) 229-233.

25) H. W. King: J. Mater. Sci. 1 (1966) 79-90.

26) M. Kiritani, T. Endoh, K. Hamada, T. Yoshiie, A. Okada, S. Kojima, Y. Satoh and H. Kayano: J. Nucl. Mater. 179-181 (1991) 1104-1107.

27) H. Okazawa, T. Yoshiie, T. Ishizai, K. Sato, Q. Xu, Y. Satoh, Y. Ohkubo and Y. Kawase: J. Nucl. Mater. 329-333 (2004) 967-970.

28) K. Ono, K. Arakawa and R. C. Birtcher: Nucl. Inst. Method. B 206 (2003) 114-117.

29) I. Mukouda and Y. Shimomura: Mater. Res. Soc. Symp. 650 (2001) R3.11.1-R3.11.6.

30) M. Kiritani: J. Nucl. Mater. 169 (1989) 89-94.

31) T. Okita, T. Sato, N. Sekimura, F. A. Garner and L. R. Greenwood: J. Nucl. Mater. 307-311 (2002) 322-326.

32) S. Yanagita, Q. Xu and T. Yoshiie: Effects of Radiation on mterials, 19th Int. Symp. ASTM STP, 1366, 2000, 516-524.

33) S. Yanagita, T. Yoshiie and H. Ino: J. Jpn. Inst. Metals 64 (2000) 115124.

34) S. Yanagita, Q. Xu, T. Yoshiie and H. Ino: Mater. Trans. 43 (2002) 1663-1669.

35) M. Kiritani, T. Yoshiie, M. Iseki, S. Kojima, K. Hamada, M. Horiki, Y. Kizuka, H. Inoue, T. Tada and Y. Ogasawara: J. Nucl. Mater. 212-215
(1994) 241-245.

36) M. Kiritani: J. Mucl. Mater. 160 (1988) 135-141.

37) M. Soroi, R. Kitamura, T. Mitsugi, T. Sekine and T. Aoyama: JNC Technical Review 21 Supplement (2003) 111-120.

38) M. Narui, H. Kurishita, H. Kayano, T. Sagawa, N. Yoshida and M. Kiritani: J. Nucl. Mater. 212-215 (1994) 1665-1670.

39) T. Muraga, S. Ohnuki, F. A. Garner and S. J. Zinkle: J. Nucl. Mater. 258-263 (1998) 130-139.

40) T. Muroga, S. J. Zinkle, A. L. Qualls and H. Watanabe: J. Nucl. Mater. 299 (2001) 148-156.

41) N. Yoshida, Q. Xu, H. Watanabe, Y. Miyamoto and T. Muroga: J. Nucl. Mater. 212-215 (1994) 471-475.

42) T. Yoshiie and M. Kiritani: J. Nucl. Mater. 191-194 (1992) 1088.

43) Y. Nagai, M. Hasegawa, Z. Tang, A. Hempel, K. Yubuys, T. Shimamura, Y. Kawazoe, A. Kawai and F. Kano: Phys. Rev. B 61 (2000) 6574-6578.

44) Q. Xu and T. Yoshiie: to be published.

45) T. Onitsuka, M. Takenaka, H. Abe, E. Kuramoto, H. Ohkubo, Y. Nagai and M. Hasegawa: Mater. Sci. Forum 445-446 (2004) 168-170.

46) H. A. Wriedt and L. S. Darken: Trans. Metall. Soc. AIME 218 (1960) 30-36.

47) G. J. Ackland, D. J. Bacon, A. F. Calder and T. Harry: Philos. Mag. A (1997) 713-732

48) F. Maury, N. Lorenzelli, M. H. Mathon, C. H. de Novion and P. Lagrde: J. Phys.: Condens. Matter. 6 (1994) 569-588.

49) C. Domain and C. S. Becquart: Phys. Rev. B 65 (2001) 024103-1-14. 